\title{
Component of Variability for Protein Content and Yield Related Traits in Recombinant Inbred Lines (RILs) Population of Bread Wheat (Triticum aestivum L. Em. Thell) in Normal and Late Sown Environments
}

\author{
Pinki*, Vikram Singh and Neha Rohila \\ College of Agriculture, Department of Genetics and Plant Breeding, CCS Haryana \\ Agricultural University, Hisar-125004, Haryana, India
}

*Corresponding author

\section{A B S T R A C T}

\begin{tabular}{|c|}
\hline Keywords \\
\hline $\begin{array}{l}\text { Yield and yield related } \\
\text { traits, Protein content, } \\
\text { Heritability, Genetic } \\
\text { advance, PCV, GCV }\end{array}$ \\
\hline Article Info \\
\hline $\begin{array}{l}\text { Accepted: } \\
12 \text { March } 2018 \\
\text { Available Online: } \\
10 \text { April } 2018\end{array}$ \\
\hline
\end{tabular}

Keywords

Yield and yield related

advance, PCV, GCV

105 RILs including the parents were evaluated for Genetic variation for protein content with yield related traits in Recombinant Inbred Lines (RILs) population of bread wheat in two field experiments i.e. normal sown and late sown. Analysis of variance revealed considerable amount of variability among RILs for all the characters in both experiments. A perusal for coefficient of variation across the two experiments revealed high GCV and PCV for flag leaf area, grain yield and biological yield. High genetic advance was observed for flag leaf area, grain yield and biological yield. High level of broad sense heritability $\left(h^{2} b\right)$ was recorded for flag leaf area, days to $50 \%$ flowering and 1000 grain weight.

\section{Introduction}

Wheat (Triticum aestivum L. Em. Thell) is the second most important cereal in India after rice. Due to its adaptation to wide agroclimatic conditions, it is most widely cultivated cereal crop and is stable food for a large proportion of world (Gupta et al., 2009). Though wheat is a cool season crop, many of the wheat growing areas are subjected to heat stress of development, which cause a reduction in yield (Kaushik et al., 2013). Grain yield, a main economic trait, is the sum total of the component characters. Thus the selection based on grain yield is usually not very successful but the one based on its component characters could be very effective. Proteins are the most important components of wheat grains governing end -use quality. Variation in both protein content and composition significantly modify flour quality for bread-making (Branlard et al., 2001). The knowledge of heritability helps in predicting the behavior of the succeeding generation and making desirable selections. Morphological traits have been studied for determine a suitable selection criterion for wheat breeding (Marti et al., 2007). Therefore, there is enough scope to increase genetic gain in yield through suitable changes in harvest index, coupled to 
an increase in total biomass. The present study was done in a RIL population of wheat to study the variability parameters of protein content and yield related traits.

\section{Materials and Methods}

The experimental material for the present investigation consisted of $103\left(\mathrm{~F}_{12}\right)$ RIL lines descended from a cross between HUW- 510/ WH-730 of bread wheat genotypes. All the RILs along with parents were sown in Randomized Block Design with 3 replications. Each plot consists 2 rows of 2 meter length with a spacing of $20 \mathrm{~cm}$ between the rows. The experimental material was sown during Rabi 2013 on two dates of sowing i.e. $\left(1^{\text {st }}\right.$ week of Nov.) and ( $1^{\text {st }}$ week of Dec.) at the wheat research area of Dept. Genetics and Plant Breeding, CCS, HAU, Hisar. All the recommended cultural practices were adopted to raise a good crop. p. Data were recorded on five randomly selected plants in each replication on characters like days to $50 \%$ heading, plant height $(\mathrm{cm})$, peduncle length $(\mathrm{cm})$, flag leaf area $(\mathrm{cm} 2)$, harvest index $(\%)$, 1000 grain weight (g) and grain protein content $(\%)$. Biological yield and grain yield which were measured on the basis of $1 / 2 \mathrm{~m}$ row length. The pedigree of parents and their ancestors of the RIL population are presented in Table 1. The Pedigree of parents and their ancestors of the RIL population are presented in Table 1.

\section{Results and Discussion}

Highly significant differences were revealed for all yield related traits and protein content in the RIL population evaluated across two environments (Table 2 and 3). Thus availability of significant genetic variability regarding all yield related traits and protein content in the population indicates that selection may be conducted with respect to these traits in the direction of increase or reductions.
The mean and range revealed a large variation for all the traits studied. The mean across all RILs in two environment (NS and LS) for all yield related traits and protein content were: $274.70 \mathrm{~g}, \quad 85.33 \mathrm{~g}, \quad 44.31 \mathrm{~g}, \quad 94.44$ days, $115.33 \mathrm{~cm}, 42.42 \mathrm{~cm}, 37.61 \mathrm{~cm}^{2}, 31.10 \%$ and $10.42 \%$ for biological yield, grain yield, 1000 grain weight, days to $50 \%$ flowering, plant height, peduncle length, flag leaf area harvest index and protein content in normal sown experiment and mean across all RILs in late sown experiment were: $224.78 \mathrm{~g}, 67.04 \mathrm{~g}$, $40.89 \mathrm{~g}, \quad 83.40$ days, $99.09 \mathrm{~cm}, 37.30 \mathrm{~cm}$, $34.33 \mathrm{~cm}^{2}, 30.11 \%$ and $10.03 \%$ for all above traits studied. The mean of RILS ranged from 135.33 to $389.33 ; 38.67$ to $137.00 ; 33.60$ to $54.33 ; 72.33$ to $121.00 ; 59.23$ to $144.90 ; 20.77$ to $58.60 ; 20.33$ to $60.37 ; 20.17$ to 39.93 and 6.71 to 14.88 for biological yield, grain yield, 1000 grain weight, days to $50 \%$ flowering, plant height, peduncle length, flag leaf area harvest index and protein content respectively (Table 4 and 5). The range for biological yield agrees with the reports of Tiwari et al., (2007). However, a different range for biological yield was reported by Kumar et al., (2013). The range for grain yield also agrees with the reports of Tiwari et al., (2007). However, a different range for grain yield was reported by Fellahi et al., 2013 (10.52-26.61). The discrepancy in range values may be attributed to different types of population, and environment and gene interaction $(\mathrm{G} \times \mathrm{E})$.

Similar range for days to $50 \%$ flowering was reported by Ali et al., 2007. However a different range for this trait was reported by Fellahi et al., (2013) (131.33-140.00). Such discrepancy may be explained due to different types of genetic material and environment used for evaluation. A similar range for plant height was reported by Majumder et al., (2008). On the contrary a lower range (63.2099.78) was reported by Fellahi et al., (2013). Similar range for peduncle length had been reported by other workers (Fellahi et al., 2013). While different range for peduncle 
length was reported by Kahrizi et al., (2010) (7.17-15.73). A similar range for flag leaf area was reported by Fellahi et al., (2013). On the contrary a lower range (13.77-21.67) was reported by Kahrizi et al., (2010). The range for harvest index agrees with the reports of Choudhary et al., (2012). While a wide range of harvest index (15.287-32.63) was observed by other workers (Majumder et al., 2008). A range for protein content was in close agreement with those of Szekely et al., (2010), Yagdi et al., (2009) and Choudhary et al., (2012).

The assessment of heritable and non-heritable components in the total variability observed is indispensable in adapting suitable breeding procedure. The heritable portion of all observed variation can be ascertained by studying the components of variation such as GCV, PCV, heritability and predicted genetic advance.

High GCV and PCV values were recorded for flag leaf area, grain yield and biological yield. These results are consistent with earlier reports (Kalimullah et al., 2012; Bhushan et al., 2013; Choudhary et al., 2012; Majunder $e t$ al., 2008; Tripathi et al., (2011). Low GCV and PCV values were recorded for days to 50\% flowering, 1000 grain weight and HI. Almost similar findings were reported by
Fellahi et al., (2013) and Tahmasebi et al., (2013). The remaining traits such as plant height, peduncle length and protein content exhibited intermediate level of GCV and PCV.

Results obtained for GCV and PCV in present investigation has revealed that there is possibility of improvement genetic improvement for majority of these characters.

It is interesting to note that the differences between GCV and PCV values were minimum which show least influence of environment on the traits namely, days to 50\% flowering, flag leaf area, harvest index (HI) and 1000 grain weight. On the other hand a substantial contribution of environment was observed on biological yield, grain yield and plant height.

High broad sense heritability was recorded for flag leaf area, Days to $50 \%$ flowering followed by 1000 grain weight in both the experiments. It was moderate for $\mathrm{HI}$ and biological yield in normal sown experiment, while in the late sown experiment besides these traits peduncle length and protein content exhibited moderate heritability. Low heritability was recorded plant height, peduncle length and protein content in normal sown experiment, while in late sown experiment plant height and grain yield exhibited low heritability.

Table.1 Pedigree of parents and their ancestors of the WH-730 $\times$ HUW-510 RIL population

\begin{tabular}{|l|l|}
\hline $\begin{array}{l}\text { Genotypes } \\
\text { Parents }\end{array}$ & Pedigree \\
\hline WH-730 & CPAN2092/Improved Lok-1 \\
\hline Ancestors & S308/S331 \\
\hline Lok-1 & II54388/AN/3/YT54/N10B/LR64 \\
\hline S308 (Sonalika) & HD2278/HUW234/DL230-16 \\
\hline Parents & HUW12*2/CPAN1666/HUW12 \\
\hline HUW-510 & NP876/CIAN066 \\
\hline HuW-234 & HD2119/247 \\
\hline HUW-12
\end{tabular}

Component of variability were determined by the appropriate statistical methods. 
Table.2 ANOVA for yield related traits and protein content in bread wheat under normal sown (NS) experiment of Rabi season, 2013-14

\begin{tabular}{|c|c|c|c|c|c|c|c|c|c|c|c|c|c|c|c|c|c|c|c|}
\hline \multirow{2}{*}{$\begin{array}{l}\text { Traits } \\
\text { Source } \\
\text { Replication } \\
\end{array}$} & \multirow{2}{*}{$\begin{array}{l}\text { D.f } \\
2 \\
2\end{array}$} & \multicolumn{2}{|c|}{$\begin{array}{l}\text { Days to } 50 \% \\
\text { flowering } \\
\text { (MS) F (cal) }\end{array}$} & \multicolumn{2}{|c|}{$\begin{array}{l}\text { Plant } \\
\text { height (cm) } \\
(\text { MS) F (cal) }\end{array}$} & \multicolumn{2}{|c|}{$\begin{array}{l}\text { Peduncle length } \\
\text { (cm) } \\
(\mathrm{MS}) \quad \text { F (cal) }\end{array}$} & \multicolumn{2}{|c|}{$\begin{array}{l}\text { Flag leaf area } \\
\left(\mathrm{cm}^{2}\right) \\
(\mathrm{MS}) \quad \mathbf{F}(\mathrm{cal})\end{array}$} & $\begin{array}{l}\text { Biological y } \\
\text { m row lengt } \\
\text { (MS) } \\
\text { (cal) }\end{array}$ & $\begin{array}{l}\text { Id } / 1 / 2 \\
(\mathrm{~g}) \\
\mathrm{F}\end{array}$ & \multicolumn{2}{|c|}{$\begin{array}{l}\text { Grain yield } / 1 / 2 \mathrm{~m} \\
\text { row length }(\mathrm{g}) \\
(\mathrm{MS}) \quad \mathrm{F}(\mathrm{cal})\end{array}$} & \multicolumn{2}{|c|}{$\begin{array}{l}\text { Harvest Index } \\
(\%) \\
\left(\begin{array}{ll}\text { MS) } & \text { F (cal) }\end{array}\right.\end{array}$} & \multicolumn{2}{|c|}{$\begin{array}{l}1000 \text { Grain } \\
\text { weight (g) } \\
\text { (MS) F (cal) }\end{array}$} & \multicolumn{2}{|c|}{$\begin{array}{l}\text { Protein content } \\
(\%) \\
\begin{array}{l}\text { MS) } \\
\text { (cal) }\end{array}\end{array}$} \\
\hline & & 8.35 & 2.99 & 1.90 & 0.70 & 2.11 & 2.59 & 1.13 & 0.50 & 16.19 & 1.839 & 4.35 & 0.57 & 4.17 & 1.93 & 1.14 & 4.97 & 0.12 & 1.04 \\
\hline Genotypes & 104 & $197.37 * *$ & 70.79 & $721.65^{* *}$ & 244.22 & $137.52 * *$ & 156.82 & $218.69 * *$ & 97.19 & $7316.02 * *$ & 777.73 & $1137.99 *$ & 136.34 & $49.03 * *$ & 22.50 & $53.03 * *$ & 230.17 & $8.22 * *$ & 67.88 \\
\hline RILs & 102 & $195.77 * *$ & 71.92 & $670.81 * *$ & 243.50 & $130.31 * *$ & 163.74 & $216.81 * *$ & 99.45 & $6864.94 * *$ & 472.74 & $1,047.47 * *$ & 134.53 & $48.01 * *$ & 21.78 & $53.29 * *$ & 230.14 & $7.49 * *$ & 64.84 \\
\hline Parents & 1 & $140.17 * *$ & 50.24 & $266.67 * *$ & 97.68 & 1.402 & 1.71 & $517.08 * *$ & 229.81 & $7072.67 * *$ & 802.80 & 6.00 & 0.78 & $64.03 * *$ & 29.51 & 0.167 & 0.73 & $32.854 * *$ & 298.67 \\
\hline $\begin{array}{l}\text { RILs Vs } \\
\text { Parents }\end{array}$ & 1 & $418.47 * *$ & 149.99 & $6406.31 * *$ & 196.64 & 1014.26 & 0.30 & $111.54 * *$ & 49.57 & $53629.53 * *$ & 562.74 & $11503.02 * *$ & 277.29 & $166.15^{* * *}$ & 49.63 & $211.45^{* * *}$ & 333.30 & $58.05^{* * *}$ & 28.73 \\
\hline Error & 208 & 2.79 & & 58.74 & & 10.58 & & 2.25 & & 498.82 & & 97.80 & & 2.74 & & 1.53 & & 0.63 & \\
\hline CV (\%) & & 1.77 & & 6.61 & & 7.66 & & 3.99 & & 8.13 & & 11.60 & & 5.34 & & 2.80 & & 7.58 & \\
\hline
\end{tabular}

* - Significant at 5\%

** - Significant at $1 \%$

Table.3 ANOVA for yield related traits and protein content in bread wheat under late sown (LS) experiment of Rabi season, 2013-14

\begin{tabular}{|c|c|c|c|c|c|c|c|c|c|c|c|c|c|c|c|c|c|c|c|}
\hline \multirow{2}{*}{$\begin{array}{l}\quad \text { Traits } \\
\text { Source } \\
\text { Replication }\end{array}$} & \multirow{2}{*}{$\begin{array}{l}\text { D.f } \\
2\end{array}$} & \multicolumn{2}{|c|}{$\begin{array}{l}\text { Days to } 50 \% \\
\text { flowering } \\
\text { (MS) F } \\
\text { (cal) }\end{array}$} & \multicolumn{2}{|c|}{$\begin{array}{l}\text { Plant } \\
\text { height }(\mathrm{cm}) \\
(\text { MS) F (cal) }\end{array}$} & \multicolumn{2}{|c|}{$\begin{array}{l}\text { Peduncle length } \\
\text { (cm) } \\
\text { ( MS) } \quad \text { F (cal) }\end{array}$} & \multicolumn{2}{|c|}{$\begin{array}{l}\text { Flag leaf area } \\
\left(\mathrm{cm}^{2}\right) \\
(\mathrm{MS}) \quad \mathrm{F}(\mathrm{cal})\end{array}$} & \multicolumn{2}{|c|}{$\begin{array}{l}\text { Biological yield } / \mathbf{1} / 2 \\
\text { m row length }(\mathrm{g}) \\
\text { ( MS) } \quad \text { F (cal) }\end{array}$} & \multicolumn{2}{|c|}{$\begin{array}{l}\text { Grain yield } / 1 / 2 \mathrm{~m} \\
\text { row length (g) } \\
(\mathrm{MS}) \quad \mathrm{F}(\mathrm{cal})\end{array}$} & \multicolumn{2}{|c|}{$\begin{array}{l}\text { Harvest Index } \\
(\%) \\
(\text { MS) } \quad \text { F (cal) }\end{array}$} & \multicolumn{2}{|c|}{$\begin{array}{l}1000 \text { Grain } \\
\text { weight (g) } \\
\text { ( MS) F (cal) }\end{array}$} & \multicolumn{2}{|c|}{ Protein content $(\%)$} \\
\hline & & 34.84 & 8.81 & 1.95 & 1.18 & 0.51 & 0.83 & 0.63 & 0.38 & 8.47 & 1.64 & 4.52 & 1.31 & 0.38 & 1.01 & 0.00 & 0.00 & 0.00 & 0.32 \\
\hline Genotypes & 104 & $155.56 * *$ & 39.35 & $627.59 * *$ & 348.25 & $122.61 * *$ & 189.90 & $124.55 * *$ & 73.72 & $6221.61 * *$ & $1,136.46$ & $555.49 * *$ & 149.90 & $59.44 * *$ & 149.65 & $39.40 * *$ & 119.77 & $7.60 * *$ & $21,477.97$ \\
\hline RILs & 102 & $156.73 * *$ & 39.99 & $575.14 * *$ & 342.55 & $118.59 * *$ & 193.78 & $123.39 * *$ & 73.21 & $5,879.18^{* *}$ & $1,131.99$ & $442.62 * *$ & 152.37 & $54.93 * *$ & 145.07 & $39.29 * *$ & 120.80 & $7.04 * *$ & $21,110.97$ \\
\hline Parents & 1 & $54.00^{* *}$ & 13.67 & 0.54 & 0.33 & $9.13 * *$ & 14.97 & $366.60 * *$ & 216.92 & $9,126.00 * *$ & 1761.78 & $4,320.17 * *$ & 1255.86 & $228.17 * *$ & 600.45 & $7.94 * *$ & 24.06 & $13.02 * *$ & 651.00 \\
\hline $\begin{array}{l}\text { RILs Vs } \\
\text { Parents }\end{array}$ & 1 & $137.22 * *$ & 34.74 & $6604.54 * *$ & 708.39 & $646.13 * *$ & 4.46 & 0.62 & 0.37 & $38.25^{* *}$ & 548.00 & $8303.55^{* *}$ & 1225.5 & $350.73 * *$ & 56.66 & $82.02 * *$ & 89.94 & $59.30^{* * *}$ & 50.5 \\
\hline Error & 208 & 3.95 & & 53.93 & & 6.81 & & 1.69 & & 345.63 & & 42.73 & & 3.55 & & 0.82 & & 0.52 & \\
\hline CV (\%) & & 2.38 & & 7.41 & & 6.10 & & 3.79 & & 8.37 & & 9.86 & & 6.31 & & 2.23 & & 7.18 & \\
\hline
\end{tabular}

* - Significant at 5\%

** - Significant at $1 \%$ 
Table.4 Mean and range for yield related traits and protein content in the HUW-510 $\times$ WH-730 RIL population of bread wheat evaluated under normal sown (NS)

experiment of Rabi season, 2013-14

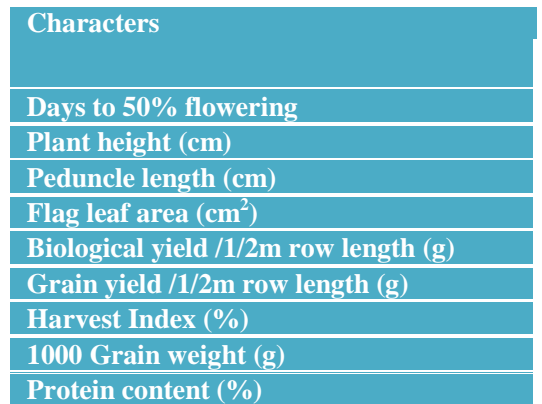

\begin{tabular}{|l|l|l|l|l|}
\hline \multirow{2}{*}{ Mean \pm S.E $(\mathrm{m})$} & \multicolumn{3}{|l|}{ Range of RILs } & Range of parents \\
\cline { 2 - 5 } & Minimum & Maximum & Minimum & Maximum \\
\hline $94.44 \pm 0.96$ & $82.00(76)$ & $121.00(53)$ & $81.33(104)$ & $91.00(105)$ \\
\hline $115.93 \pm 0.95$ & $62.63(30)$ & $144.90(100)$ & $99.90(104)$ & $113.23(105)$ \\
\hline $42.42 \pm 0.52$ & $24.33(30)$ & $58.60(20)$ & $41.73(105)$ & $42.70(104)$ \\
\hline $37.61 \pm 0.87$ & $20.33(40)$ & $60.37(29)$ & $32.60(104)$ & $51.17(105)$ \\
\hline $274.70 \pm 2.19$ & $139.33(36)$ & $389.33(52)$ & $217.67(104)$ & $286.33(105)$ \\
\hline $85.33 \pm 1.60$ & $40.67(36)$ & $137.00(51)$ & $65.67(104)$ & $67.67(105)$ \\
\hline $31.10 \pm 0.85$ & $20.17(23)$ & $39.93(8)$ & $23.63(105)$ & $30.17(104)$ \\
\hline $44.31 \pm 0.27$ & $34.53(36)$ & $54.33(51)$ & $40.60(105)$ & $40.93(104)$ \\
\hline $10.42 \pm 0.19$ & $6.93(45)$ & $14.88(36)$ & $8.80(104)$ & $13.48(105)$ \\
\hline
\end{tabular}

HUW-510 = Parent No. 104 WH-730 = Parent No. 105

Table.5 Mean and range for yield related traits and protein content in the HUW-510 $\times$ WH-730 RIL population of bread wheat evaluated under late sown (LS)

experiment of Rabi season, 2013-14

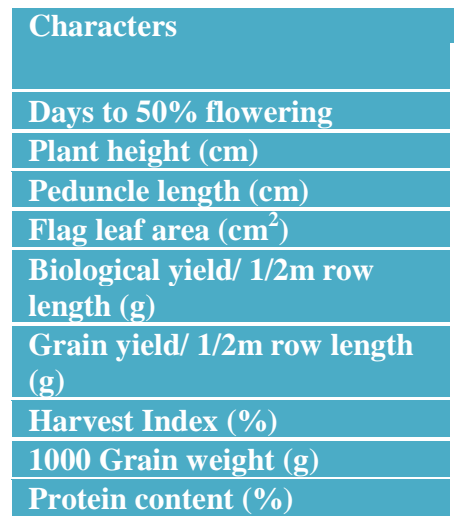

\begin{tabular}{|l|}
\hline Mean \pm S.E $(\mathbf{m})$ \\
\hline $83.40 \pm 1.15$ \\
\hline $99.09 \pm 0.74$ \\
\hline $37.30 \pm 0.45$ \\
\hline $34.33 \pm 0.75$ \\
\hline $224.79 \pm 1.31$ \\
\hline $67.04 \pm 1.07$ \\
\hline $30.11 \pm 0.35$ \\
\hline $40.89 \pm 0.33$ \\
\hline $10.03 \pm 0.01$ \\
\hline
\end{tabular}

\begin{tabular}{|l|l|l|l|}
\hline Range of RILs & \multicolumn{2}{|l|}{ Range of parents } \\
\hline Minimum & Maximum & Minimum & Maximum \\
\hline $72.33(76)$ & $106.00(8)$ & $75.67(104)$ & $81.67(105)$ \\
\hline $59.23(36)$ & $139.17(94)$ & $84.97(104)$ & $85.57(105)$ \\
\hline $20.77(60)$ & $52.60(20)$ & $35.40(105)$ & $37.87(104)$ \\
\hline $21.47(40)$ & $51.77(54)$ & $26.83(104)$ & $42.47(105)$ \\
\hline $135.33(36)$ & $319.33(23)$ & $207.33(104)$ & $285.33(105)$ \\
\hline $38.67(47)$ & $99.33(55)$ & $63.00(105)$ & $71.33(104)$ \\
\hline $21.47(57)$ & $45.83(51)$ & $22.07(105)$ & $34.40(104)$ \\
\hline $33.60(99)$ & $50.13(52)$ & $37.53(105)$ & $39.83(104)$ \\
\hline $6.71(90)$ & $13.97(36)$ & $9.55(104)$ & $12.50(105)$ \\
\hline
\end{tabular}

HUW-510 = Parent No. 104 WH-730 = Parent No. 105

Table.6 Components of variance and variability for yield related traits and protein content in the HUW-510 $\times$ WH-730 RIL population of bread wheat evaluated under normal sown (NS)

experiment of Rabi season, 2013-14

\begin{tabular}{|c|c|c|c|c|c|c|c|c|c|}
\hline & $\begin{array}{c}\text { Days to } \\
50 \% \\
\text { flowering } \\
\end{array}$ & $\begin{array}{l}\text { Plant } \\
\text { height } \\
(\mathrm{cm})\end{array}$ & $\begin{array}{c}\text { Peduncle } \\
\text { length } \\
\text { (cm) }\end{array}$ & $\begin{array}{l}\text { Flag } \\
\text { leaf } \\
\text { area } \\
\left(\mathrm{cm}^{2}\right) \\
\end{array}$ & $\begin{array}{c}\text { Biological } \\
\text { yield } / 1 / 2 \mathrm{~m} \\
\text { row length } \\
\text { (g) }\end{array}$ & $\begin{array}{l}\text { Grain yield } \\
/ 1 / 2 \mathrm{~m} \text { row } \\
\text { length(g) }\end{array}$ & $\begin{array}{c}\text { Harvest } \\
\text { Index } \\
(\%)\end{array}$ & $\begin{array}{c}\begin{array}{c}1000 \\
\text { Grain } \\
\text { weight }\end{array} \\
(\mathrm{g})\end{array}$ & $\begin{array}{c}\text { Protein } \\
\text { content } \\
(\%)\end{array}$ \\
\hline $\begin{array}{l}\text { Genotypic variance } \\
\sigma^{2} g\end{array}$ & 64.86 & 220.97 & 42.33 & 72.15 & 2272.40 & 346.73 & 15.52 & 17.59 & 2.53 \\
\hline $\begin{array}{l}\text { Phenotypic variance } \\
\sigma^{2} p\end{array}$ & 67.65 & 279.71 & 52.91 & 74.40 & 2771.22 & 444.53 & 18.26 & 19.12 & 3.16 \\
\hline $\begin{array}{l}\text { Variance due to } \\
\text { envir. } \sigma^{2} \mathrm{e}\end{array}$ & 2.79 & 58.74 & 10.58 & 2.25 & 498.82 & 97.80 & 2.74 & 1.53 & 0.63 \\
\hline $\begin{array}{l}\text { Broad sense } \\
\text { heritability } \mathbf{h}^{2}(\mathrm{bs})\end{array}$ & 0.96 & 0.79 & 0.80 & 0.97 & 0.82 & 0.78 & 0.85 & 0.92 & 0.80 \\
\hline Genotypic CV (\%) & 8.53 & 12.82 & 15.34 & 22.58 & 17.35 & 21.82 & 12.67 & 9.47 & 15.26 \\
\hline Phenotypic CV (\%) & 8.71 & 14.42 & 17.14 & 22.93 & 19.16 & 24.70 & 13.73 & 9.86 & 17.08 \\
\hline $\begin{array}{l}\text { Genetic Advance \% } \\
\text { of mean }\end{array}$ & 17.20 & 23.47 & 28.24 & 45.81 & 32.37 & 39.69 & 24.05 & 18.69 & 28.12 \\
\hline
\end{tabular}


Table.7 Components of variance and variability for yield related traits and protein content in the HUW-510 $\times$ WH-730 RIL population of bread wheat evaluated under late sown (LS) experiment of Rabi season, 2013-14

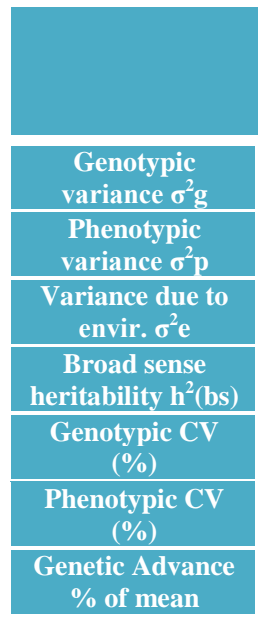

\begin{tabular}{|c|c|c|c|c|c|c|c|c|}
\hline $\begin{array}{c}\text { Days to } \\
50 \% \\
\text { flowering }\end{array}$ & $\begin{array}{l}\text { Plant } \\
\text { height } \\
\text { (cm) }\end{array}$ & $\begin{array}{l}\text { Peduncle } \\
\text { length } \\
\text { (cm) }\end{array}$ & $\begin{array}{c}\text { Flag leaf } \\
\text { area } \\
\left(\mathrm{cm}^{2}\right)\end{array}$ & $\begin{array}{c}\text { Biological } \\
\text { yield } / 1 / 2 \mathrm{~m} \\
\text { row length } \\
\text { (g) }\end{array}$ & $\begin{array}{c}\text { Grain } \\
\text { yield/1/2m } \\
\text { row } \\
\text { length }(\mathrm{g})\end{array}$ & $\begin{array}{l}\text { Harvest } \\
\text { Index } \\
(\%)\end{array}$ & $\begin{array}{c}1000 \\
\text { Grain } \\
\text { weight } \\
\text { (g) }\end{array}$ & $\begin{array}{c}\text { Protein } \\
\text { content } \\
(\%)\end{array}$ \\
\hline 50.53 & 191.22 & 38.60 & 40.95 & 1958.66 & 170.92 & 18.63 & 12.86 & 2.36 \\
\hline 54.49 & 245.15 & 45.41 & 42.64 & 2304.23 & 213.65 & 22.18 & 13.68 & 2.88 \\
\hline 3.95 & 53.93 & 6.81 & 1.69 & 345.63 & 42.73 & 3.55 & 0.82 & 0.52 \\
\hline 0.93 & 0.78 & 0.85 & 0.96 & 0.85 & 0.80 & 0.84 & 0.94 & 0.82 \\
\hline 8.52 & 13.96 & 16.66 & 18.64 & 19.69 & 19.50 & 14.34 & 8.77 & 15.33 \\
\hline 8.85 & 15.80 & 18.07 & 19.02 & 21.35 & 21.81 & 15.64 & 9.05 & 16.95 \\
\hline 16.91 & 25.39 & 31.64 & 37.63 & 37.40 & 35.93 & 27.07 & 17.51 & 28.61 \\
\hline
\end{tabular}

Table.8 Frequency of transgressive RILs for all yield related traits and protein content in the RIL population of wheat in the normal sown (NS) experiment of Rabi season, 2013-14

\begin{tabular}{|c|c|c|c|c|c|c|c|c|c|}
\hline Characters & $\begin{array}{l}\text { Days to } 50 \% \\
\text { flowering }\end{array}$ & $\begin{array}{l}\text { Plant } \\
\text { height } \\
(\mathrm{cm})\end{array}$ & $\begin{array}{l}\text { Peduncle } \\
\text { length }(\mathrm{cm})\end{array}$ & $\begin{array}{l}\text { Flag } \\
\text { leaf } \\
\text { area } \\
\left(\mathrm{cm}^{2}\right)\end{array}$ & $\begin{array}{l}\text { Biological yield } \\
/ 1 / 2 \mathrm{~m} \text { row } \\
\text { length }(\mathrm{g})\end{array}$ & $\begin{array}{l}\text { Grain } \\
\text { yield } \\
/ 1 / 2 \mathrm{~m} \\
\text { row } \\
\text { length (g) }\end{array}$ & $\begin{array}{l}\text { HI } \\
(\%)\end{array}$ & $\begin{array}{l}\mathbf{1 0 0 0} \\
\text { Grain } \\
\text { weight } \\
\text { (g) }\end{array}$ & $\begin{array}{l}\text { Protein } \\
\text { content } \\
(\%)\end{array}$ \\
\hline $\begin{array}{l}+ \text { Ve } \\
\text { Transgressive } \\
\text { RILs }\end{array}$ & 63 & 65 & 46 & 7 & 41 & 85 & 66 & 82 & 2 \\
\hline $\begin{array}{l}\text { - Ve Transgressive } \\
\text { RILs }\end{array}$ & 0 & 12 & 49 & 28 & 11 & 13 & 4 & 11 & 15 \\
\hline $\begin{array}{l}\text { Intermediate } \\
\text { Transgressive } \\
\text { RILs }\end{array}$ & 40 & 26 & 8 & 68 & 51 & 5 & 33 & 10 & 86 \\
\hline
\end{tabular}

Heritability estimates are useful in choosing the characters for constructing the selection indices. High heritability values for traits indicated that the variation observed was mainly under genetic control and was least effected by environment. Similar results were reported by Tripathi et al., (2011), Majunder et al., (2008) and Tahmasebi et al., (2013).

It is apparent from above that the improvement of various characters, individually or simultaneously, different selection intensities are to be exercised depending on estimates of genetic components of variation and the heritability of concerned traits. However, GCV itself would not be a correct measure to know the heritable variations present and therefore, GCV should be considered together with heritability estimates to get the more reliable picture of the amount of the advance to be expected from the selections (Burton, 1952).

High genetic advance was observed for flag leaf area, grain yield and biological yield. Moderate genetic advance was recorded for protein content, plant height and peduncle length and HI and low for 1000 grain weight and days to $50 \%$ flowering (Table 6 and 7). 
The high heritability estimates would be helpful for breeding superior genotypes on the basis of phenotypic performance of quantitative characters. However, Johnson et $a l$. , (1995) reported that heritability estimates along with genetic gain were more useful than the heritability values alone for selecting the best individual. Panse (1957) further elaborated that high heritability together with high genetic advance was an indication of absence of dominance and epistatic effects. In the present study biological yield, grain yield, flag leaf area and protein content exhibited high genetic advance as per cent of mean along with high heritability estimates indicates that most likely the heritability is due to the additive gene effects and selection may be effective in early generations for these traits. Similar findings have been reported by Kalimullah et al., (2012).

High heritability along with moderate genetic advance was noticed for plant height and peduncle length suggesting predominance of additive and non-additive gene action in the expression of these traits. Therefore, these traits can be improved by mass selection. High heritability along with low genetic advance was noticed for days to $50 \%$ flowering is indicative of predominance of non-additive gene actions which could be exploited through heterosis breeding. Besides, Akanda et al., (1997) reported that high genotypic coefficient of variation along with high heritability and genetic advance provide better information than other parameters alone.

These observations are in agreement with the earlier reports of Fellahi et al., (2013), Majunder et al., (2008) and Bhushan et al., (2013). Subhashchandra et al., (2009) also reported high heritability and high genetic advance for these traits indicating the scope for their improvement through simple selection.
All yield related traits and protein content are quantitative in nature and polygenically controlled. The parents are expected to have dispersion of genes controlling a trait. To analyse this hypothesis segregants surpassing parental means both in positive and negative direction were examined for morphological traits and number of such transgressive segregants for each trait are presented in Table 8. It is evident that a number of transgressive segregants in both positive and negative direction were presented for each of the morphological traits in RIL population. Therefore, each of the parents possessed both increasing and decreasing effects, i.e. genes were dispersed between the parents.

During the course of development of RILs, there was repeated opportunity for recombination in $\mathrm{F}_{2}$ and onward generation's, leading to accumulation of gene with like effects in the resulting RILs. This is further substantiated that parental genotype exhibited a narrow range for different morphological traits but a number of transgressive segregants were present in RIL population for different yield related traits and protein content. The subsequent QTL analysis for morphological traits in this population may be pursued and characterize the parental genotypes for possessing. QTLs (genes) with increasing or decreasing effects and thus would confirm the gene dispersion of polygene governing these traits.

\section{References}

Akanda, M.A.L., Alam, M.S. and Shamsuddin, A.K. (1997). Genetic variability, correlation and path analysis in wheat. Bangladesh J. Pl. Breed. Genet. 10(1): 57-61.

Ali, S., Shah, S.M., Hassnain, A., Shah, Z. and Munir, I. (2007). Genotypic variation for yield and morphological 
traits in wheat. Sarhad J. Agric. 23: 943-946.

Bhushan, B., Bharti, S., Ojha, A., Pandey, M., Gourav, S.S., Tyagi, B.S. and Singh, G. (2013). Genetic variability, correlation coefficient and path analysis of some quantitative traits in bread wheat. $J$. Wheat Res. 5(1): 21-26.

Bishnoi, S. (2014). Molecular mapping of quantitative trait loci for yield, yield components and terminal heat tolerance in bread wheat (Triticum aestivum L.). Thesis submitted to Choudhary Charan Singh Haryana Agricultural University.

Branlard, G., Dardevet, M., Saccomano, R., Lagoutte, F. and Gourdon, J. (2001). Genetic diversity of wheat storage proteins and bread wheat quality. Euphytica. 119: 59-67.

Burton, G. W. (1952). Quantitative inheritance in grasses. Proceedings Intl. Grassld. Congress. 1: 277-283.

Choudhary, R., Jat, B.L., Anwala, R., Dhikwal, S.R. and Sharma, K.C. (2012). Studies on estimates of genetic variability and character association of yield components and protein content in pearlmillet. Forage Res. 38(2): 80-85.

Fellahi, Z., Hannachi, A., Bouzerzour, A. and Boutekrabt, A. (2013). Study of interrelationship among yield and yield related attributes by using various statistical methods in bread wheat (Triticum aestivum L.). Intl. J. Agron. Prod. 4(6): 1256-1266.

Gollen, B. (2014). Genetic analysis of grain filling components using molecular markers in RILs of bread wheat (Triticum aestivum L.). Thesis submitted to Choudhary Charan Singh Haryana Agricultural University.

Gupta, P.K., Peter, L. and, Mir, R. (2009). Marker-assisted wheat breeding present status and future possibilities. $\mathrm{Mol}$ Breed. 7: 921-935.
Johnson, B.F. Trethowan, R.G. and Sears, R.G. (1995). Genetic variability, heritability and genetic advance in bread wheat (Triticum aestivum L.) under salinity stress conditions. Agric. J. 97 (13): 29-30.

Kahrizi, D., Cheghamirza, K., Kakael, M., Mohammadi, R. and Ebadi A. (2010). Heritability and genetic gain of some morpho-physiological variables of durum wheat (Titicum turgidum var. durum). Afric. J. Biotech. 9: 4687-4691.

Kalimullah., Khan, S.J., Irfaq, M. and Rahman, H.U. (2012). Genetic variability, correlation and diversity studies in bread wheat (Triticum aestivum L.) germplasm. J. Anim and Pl. Sci. 22(2): 330-333.

Kaushik, S.K., Tomar, H.U., Dixit, A.K. and Saxena, A.K., (2013). Assessment of wheat varieties in Central India under changed climatic scenario. Wudpecker J. Agric. Res. 2 (2): $64-66$.

Majumder, D.A.N., Shamsuddin, A.J., Kabir, M. and. Hussan, L. (2008). Genetic variability, correlated response and path analysis of yield and yield contributing traits of spring wheat. J. Bangladesh Agril. Univ. 6(2): 227-234.

Mala (2013). Genetic analysis of kernel traits in bread wheat (Triticum aestivum L.). Thesis submitted to Choudhary Charan Singh Haryana Agricultural University.

Marti, J., Bort, J., Slafer, G.A. and Araus, J.L. (2007). Can wheat yield be assessed by early measurements of normalized difference vegetation index? Annal. Appl. Biol. 150: 253-257.

Panse, V.G. (1957). Genetics of quantitative characters in relation to plant breeding. Ind. J. Genet. 17: 318-328.

Subhashchandra, B., Lohithaswa, H.C., Desai, S.A., Hanchinal, R.R., Kalappanavar, I.K., Math, K.K. and, Salimath, P.M. (2009). Assessment of genetic variability and relationship between 
genetic diversity and transgressive segregation in tetraploid wheat. Karnataka J. Agric. Sci. 22 (1): 36-38.

Szekely, E., Moldovan, Y., Kadar, R. and Has, I. (2010). Research regarding to some morpho-physiological and quality traits in winter wheat collection from agricultural research and development station Turda. Res. J. Agric.sci. 42(2): 314-321.

Tahmasebi, G., Heydarnezhadian, J. and Aboughadareh, A.P. (2013). Evaluation of yield and yield components in some of promising wheat lines. Int. J. Agril. And crop Sci. 5(20): 2379-2384.
Tiwari, V. (2007). Grain filling duration as a means for increasing yield in spring wheat. Ind. J. Genet. 67(4): 365-368.

Tripathi, S.N., Marker, S., Pandey, P., Jaiswal, K.K. and Tiwari, D.K. (2011). Relationship between some morphological and physiological traits with grain yield in bread wheat (Triticum aestivum L.). Trends Appl. Sci. Res. 6(9): 1037-1045.

Yagdi, K. and Sozen, E. (2009). Heritability, variance components and correlations of yield and quality traits in durum wheat (Triticum durum desf.). Pak. J. Bot. 41(2): 753-759.

\section{How to cite this article:}

Pinki, Vikram Singh and Neha Rohila. 2018. Component of Variability for Protein Content and Yield Related Traits in Recombinant Inbred Lines (RILs) Population of Bread Wheat (Triticum aestivum L. Em. Thell) in Normal and Late Sown Environments. Int.J.Curr.Microbiol.App.Sci. 7(04): 1539-1547. doi: https://doi.org/10.20546/ijcmas.2018.704.173 